\title{
Screen-printed $p$-CdTe layers for CdS/CdTe solar cells
}

\author{
V.P. Klad'ko, P.M. Lytvyn, N.M. Osipyonok, G.S. Pekar, I.V. Prokopenko, A.F. Singaevsky \\ $V$. Lashkaryov Institute of Semiconductor Physics, NAS of Ukraine, \\ 45, prospect Nauky, 03028 Kyiv, Ukraine \\ Phone: +380 (44) 5255940; e-mail:plyt@isp.kiev.ua
}

\begin{abstract}
Correlation of the recrystallization process technological parameters with the morphology and structure of screen-printed $p$-CdTe layers intended for CdS/CdTe solar cell fabrication has been established. The optimal regimes to form layers with required characteristics have been found. As distinct from the used previously screen-printing techniques for $\mathrm{CdS} / \mathrm{CdTe}$ solar cell fabrication, CdTe layers were doped with $\mathrm{Ag}$ or $\mathrm{Au}$ not by their diffusion from the layer surface but in the course of layer preparation. For this purpose, tellurides of those metals were added into the raw paste used for CdTe screen printing. It is shown that the developed method has some advantages and allows to prepare CdTe films, structural and electrophysical parameters of which are suitable to fabricate $\mathrm{CdS} / \mathrm{CdTe}$ solar cells.
\end{abstract}

Keywords: CdTe, screen printing, crystal structure and symmetry, X-ray diffraction, solar cell.

Manuscript received 15.04.05; accepted for publication 18.05.05.

\section{Introduction}

Screen-printed polycrystalline CdTe thick layers are promising components for creating photosensitive $n$ $\mathrm{CdS} / p$-CdTe heterojunctions whose efficiency of solar energy conversion may be as high as $16 \%$ [1]. Screenprinting technologies as well as the methods for creating the raw materials used for layer preparation are rather well developed. However, the properties of the layers under consideration should comply with specified requirements: the layers have to be mechanically proof, continuous, rather low-resistivity and have to possess a good adhesion to the substrate. In addition, CdTe layers must have the $p$-type conductivity. To meet those requirements, it is necessary to develop special receipts for layer screen-printing as well as optimal regimes for layer crystallization.

It is obvious that the latter regimes are very important to provide the layer properties listed above, especially taking into account the polycrystalline nature of the layers. Such regimes are aimed at obtaining the close-packed crystalline structure with the grain sizes comparable to the layer thickness. In addition, those regimes are capable to avoid (at least, partially) "secondary", frequently undesirable processes following recrystallization, such as interdiffusion, formation of new phases and point defects, oxidation, macrostrain relaxation with formation of dislocations, etc. [2].

In this work, the correlation of the recrystallization process technological parameters with the morphology and structure of the $p$-CdTe layers is established, the optimal regimes for formation of the layers with required characteristics are found. As distinct from the screenprinting techniques for $\mathrm{CdS} / \mathrm{CdTe}$ solar cell fabrication described previously (in those techniques the $p$-type conductivity of CdTe layers was provided by $\mathrm{Cu}$ diffusion from the layer surface [3]), we have doped CdTe layers with Ag or Au during the layer formation. Toward this end, the tellurides of those metals were added into the raw paste used for CdTe screen printing. Ultimately, the intention of this work is to obtain the films with structural and electrophysical parameters feasible to fabricate $\mathrm{CdS} / \mathrm{CdTe}$ solar cells.

\section{Experimental}

Similarly to the technique for CdS layer preparation described previously [4], CdTe layers were prepared by screen-printing using the recrystallizating flux of cadmium chalcogenide group.

Commercially-available high-purity (6N) CdTe powder was mixed with distilled water as an anticoagulant and then was crushed for 24 hours in a ball mill with a chalcedony drum. The obtained paste was dried at 190 to $200{ }^{\circ} \mathrm{C}$ in the inert gas flow. Then the powder prepared was termally treated at $600{ }^{\circ} \mathrm{C}$ for $6 \mathrm{~h}$ in the inert atmosphere. After such preliminary treatment, the grain size did not exceeded $1.5 \mu \mathrm{m}$. Commercially-available $\mathrm{CdCl}_{2}$ powder of a chemical purity was dried at about $200{ }^{\circ} \mathrm{C}$ for an hour in inert atmosphere and then was crushed in an air-stream mill. 
The fraction with a grain size less than $1.5 \mu \mathrm{m}$ was selected for further use. CdTe and $\mathrm{CdCl}_{2}$ powders were mixed with an organic binder (propylene glycol) and then were treated in a special unit for preparing a homogeneous paste. The material for doping was treated similarly to CdTe powder, then was mixed with an organic binder and further was added to the paste prepared.

The humid layers CdTe prepared as described above, were consisted of separated sections of deposited paste which represented the structure of the grid for screen printing. However, for a short period of time (some minutes), those sections closed up and formed a layer of a uniform thickness. Such layers were dried in a traveling oven for $60 \mathrm{~min}$ at the temperature of 130 to $150{ }^{\circ} \mathrm{C}$ in an inert gas flow. When heating, the organic binder was partially evaporated from the layer (evaporation of the rest of the binder took place at the first stage of the final process of sintering ).

The substrates with deposited dried layers were placed in a quartz glass case whose cover had holes, and then the case was slow pulled inside the heated traveling oven with an automatic temperature control. Then, the screen-printed $\mathrm{CdTe}$ layers were sintered at various temperatures from 600 to $650^{\circ} \mathrm{C}$ for 1,3 , and 6 hours in the argon atmosphere.

After finishing the re-sintering process, the case with the substrate was cooled in the inert atmosphere (inside the furnace) to room temperature.

As distinct from previous works on screen-printed $\mathrm{CdS} / \mathrm{CdTe}$ solar cells (where $p$-type conductivity of $\mathrm{CdTe}$ layers was provided by $\mathrm{Cu}$ diffusion from the layer surface [3]), we doped CdTe with Ag or Au. Those acceptor impurities were introduced during CdTe layer formation. For this purpose, Ag and $\mathrm{Au}$ tellurides were added into the paste before the screen-printing process. It is clear that it was undesirable to use tellurides obtained by chemical reactions aided by some foreign chemical agents since they may result in the uncontrolled additional doping of CdTe layers. At the same time, the methods for direct synthesis of such tellurides described previously in the chemical literature are rather long and inconvenient when being used [5].

When developing the safe, easy-to-use and cheap method for a direct synthesis of Ag and Au tellurides, we have based on the semiconductor heat treatment under the vapour pressure of volatile matters developed in our bygone work [6]. From [6], we took the scheme of the apparatus shown in Fig. 1a.

The initial metal 1 ( $\mathrm{Ag}$ or $\mathrm{Au})$ was placed into the quartz glass crucible 2. The crucible was placed inside the quartz glass tube 3 that may be closed by the ground quartz glass cover 4 with two branch pieces 6 . Some quantity of Te powder or granules was placed at the tube bottom. The quantities both of metal and Te are not critical. The inert gas (such as Ar) flows in the tube through a thin tube 5 inserted inside the first branch piece and flows out through the second one 6. Quartz glass heat-reflecting screens were located above the crucible. The tube was inserted inside the furnace with a resistance-type heater.

During the heating the furnace whose temperature profile is shown in Fig. 1b, tellurium specimen turns to a vapour and further is condensed on the surfaces of the heat-reflecting screens and inner tube walls. The formed drops fell down in the evaporation zone and then convert into a vapour again. Such a circulation continues during the whole technological process. Note that the total pressure inside the tube is close to the atmospheric pressure.

During the whole process, the metal was surrounded by Te vapour. The temperature of the metal was set equal or higher than that required for telluride synthesis. As a result, with the operation of heating completed, the whole quantity of the metal reacted with some Te, and some quantity of telluride had formed at the crucible. Then, the furnace was cooled to the room temperature, the cover was opened, the telluride was taken out and, what is important, the whole system could be used over and over again.

By means of the method described above, we have sintered $\mathrm{Ag}$ and $\mathrm{Au}$ tellurides for their further use as materials for CdTe layer doping.

The uniformity of CdTe films surface, transformation dynamics of grain sizes, the shape of the grains, and changes in the grain boundaries in the course of annealing were monitored by the optical and atomic force microscopy (AFM) methods. The structural analysis was done by X-ray diffraction (XRD) with DRON-3M diffractometer using $\mathrm{Cu} K_{\alpha}$ radiation $(\lambda=0.1542 \mathrm{~nm})$. The photoluminescence spectra were measured at $77 \mathrm{~K}$ on excitation with nitrogen laser beam (excitation $\lambda=337 \mathrm{~nm}$ ) in order to determine characteristics of point defects and impurities.

The electrical conductivity of the layers prepared was measured with the standard four-probe van der Pauw method [7], the type of electrical conductivity was

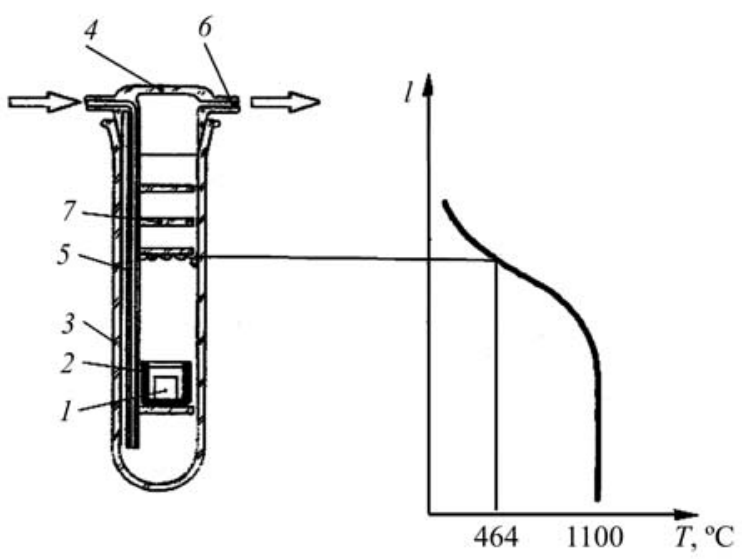

Fig. 1. Scheme of the reactor [6] used for the direct synthesis of metal tellurides (a) and the temperature profile of the furnace (b): 1 - initial metal; 2 - quartz glass crucible; 3 quartz glass tube; 4 - quartz glass cover; 5 - thin tube; 6 branch piece; 7 - heat-reflecting screen. 


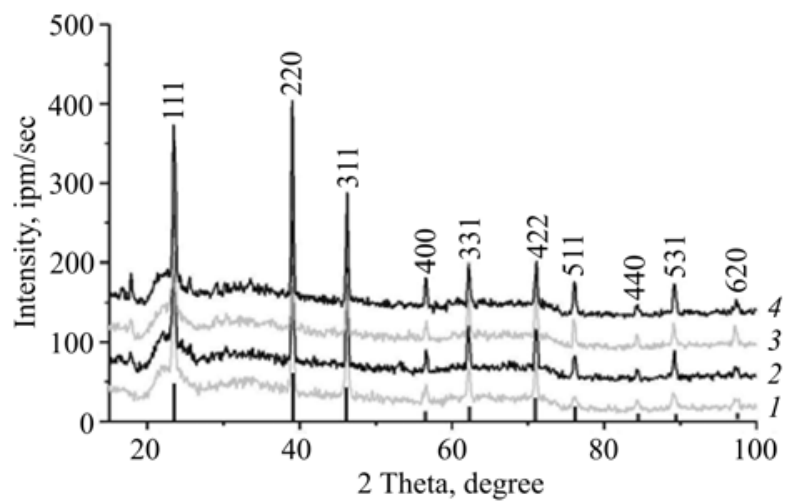

Fig. 2. X-ray diffraction patterns of CdTe layer just as result of the drying (1) and annealing at $630{ }^{\circ} \mathrm{C}$ for $1 \mathrm{~h}(2), 3$ (3), and 6 (4). Bars represent positions and intensities of the simulated X-ray pattern for a CdTe powder.

measured by the thermal probe method. Electrical contacts to CdTe layers were fabricated from $\mathrm{Ag}$ and carbon paste [8].

\section{Results and discussion}

Crystalline structure of CdTe layers was studied by a single-crystal X-ray diffractometer using $\mathrm{Cu} K_{\alpha}$ radiation. To provide the high sensitivity and improved resolution the diffractometer was supply with focusing $\mathrm{LiF}$ monochromator as well as with a system for the digital recording of the spectra. Diffraction spectra were obtained by $2 \vartheta$-scanning over the angles from 15 to 100 degrees.

We examined the wide variety of CdTe layers at different stages of the technological process of their

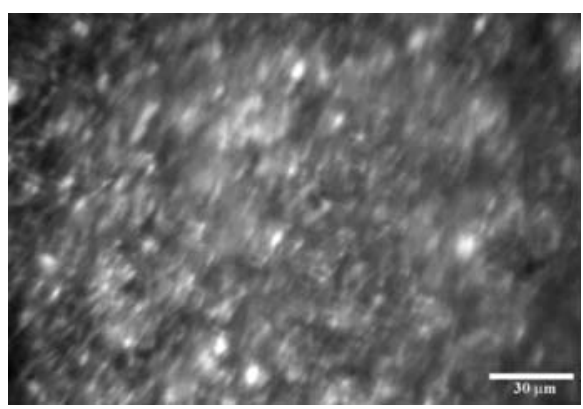

(a)

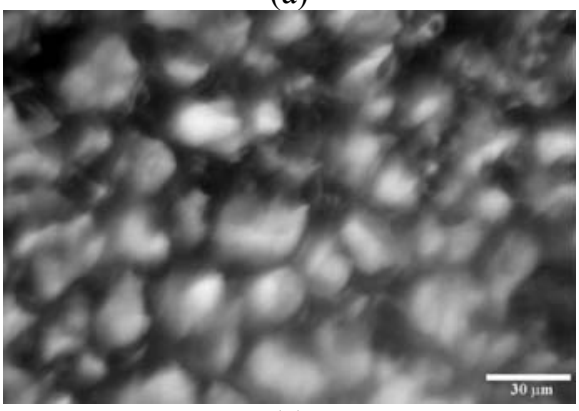

(c) fabrication as well as CdTe layers prepared under different technological conditions. Such an approach made it possible to establish those technological conditions for polycrystalline layer sintering that provide the highest level of structural perfection of the layers. It was found that the annealing temperature and duration were the main technological parameters that determined the layer perfection. It was established that the most perfect CdTe layers could be prepared at the annealing temperature from 630 to $650{ }^{\circ} \mathrm{C}$ and annealing duration from 2 to $3 \mathrm{~h}$. The examples of X-ray spectra of CdTe layers prepared at the same annealing temperature $630{ }^{\circ} \mathrm{C}$ and different annealing duration are given in Fig. 2. It follows from the data obtained that, already after paste drying, the CdTe layers do not contain extraneous phases, and the microcrystalline CdTe phase dominates (i.e., quasi-amorphous phase is absent) (Fig. 2, curve 1). Annealing at $630{ }^{\circ} \mathrm{C}$ for $1 \mathrm{~h}$ promotes the intensive crystallization process. As a result, the crystallite size increases significantly (up to about $30 \mu \mathrm{m}$ ), and the crystalline orientation (111) becomes preferable. This process manifests itself in X-ray diffraction spectra as an increase of the diffraction peak heights (when decreasing their half-width) and as a predominance of the reflection (111) peak over (220) peak (Fig. 2, curve 2). The increase in annealing duration promotes the grain coalescence, which results in the increase of the grain size (to about $1000 \mathrm{~nm}$ ) and in texture destruction (Fig. 2, curve 4).

Morphology of the layer surface was studied by the methods of optical microscopy and AFM. Fig. 3 shows the images of the layer surface before and after resintering process obtained by means of optical

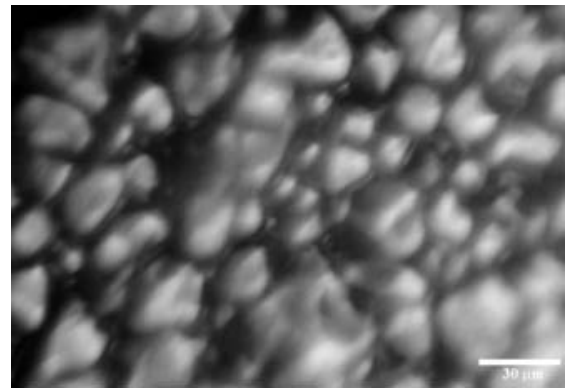

(b)

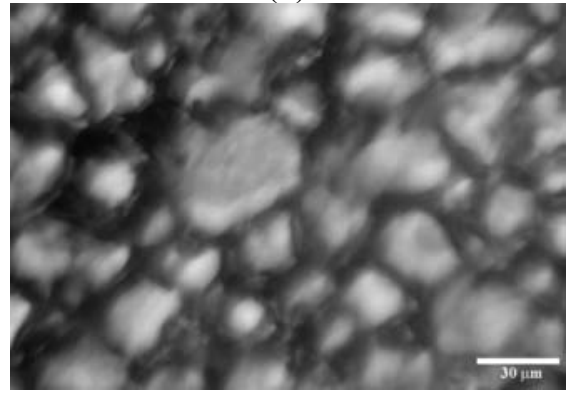

(d)

Fig. 3. Optical microscopy images of the initial surface of CdTe layers (a) and the same surface after annealing at $630^{\circ} \mathrm{C}$ for $1 \mathrm{~h}$ (b), 3 (c), and 6 (d). 
microscope. The pictures point to the significant rise in the grain sizes resulted from the layer heat treatment (Figs 3b, c, d) as well as to the absence of structural macrodefects such as pores, cavities, cracks, etc.

The changes in the grain sizes listed above correlates with the results of X-ray diffraction measurements: in consequence of one-hour annealing, the size of the grains in the layer surface increases by a factor of ten as compared with that in the dried layer but the grain size distribution is be-modal, i.e., the most of grains comprised two sizes - 10 and $30 \mu \mathrm{m}$ (Fig. 3b). If the annealing duration is increased to $3 \mathrm{~h}$ the grain size becomes almost the same (from 25 to $35 \mu \mathrm{m}$ ), and the grain boundaries become smooth (Fig. 3c). The increase in the annealing duration to $6 \mathrm{~h}$ has not resulted in the essential changes in the surface properties, although some tendency to the grain size increase was observed (Fig. 3d).

AFM investigations of the fine structure of CdTe layers were carried out by the AFM unit NanoScope IIIa Dimention $3000^{\mathrm{TM}}$ made by Digital Instruments. The measurements were performed in the Tapping mode by means of silicon probes of NSG01 type fabricated by NT-MDT company, the nominal radius of the tip apex curvature equals about $10 \mathrm{~nm}$.

The 3D AFM image of the CdTe layer upon recrystallization annealing is shown in Fig. 4. In-phase growth steps (terraces) (from 30 to $150 \mathrm{~nm}$ in width and from 3 to $6 \mathrm{~nm}$ in height) have been observed on the layer surface. They indicate the equilibrium grain growth as well as the absence of impurities in the grain bulk. The grains are divided by distinct boundaries, and the terrace growth stops mainly at those boundaries. This can be indicative of the high point defect density at the boundaries.

As known, the value of resistivity of CdTe layers and the type of electrical conductivity are the main physical

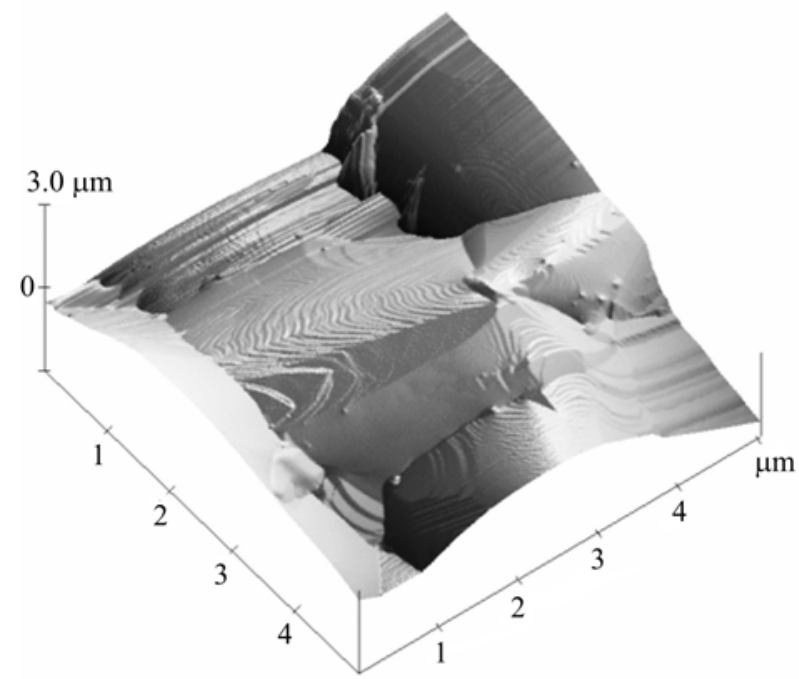

Fig. 4. $3 \mathrm{D}$-image of the fragment of $\mathrm{CdTe}$ layer surface obtained by the AFM method. The layer is annealed at $650{ }^{\circ} \mathrm{C}$ for $2 \mathrm{~h}$.

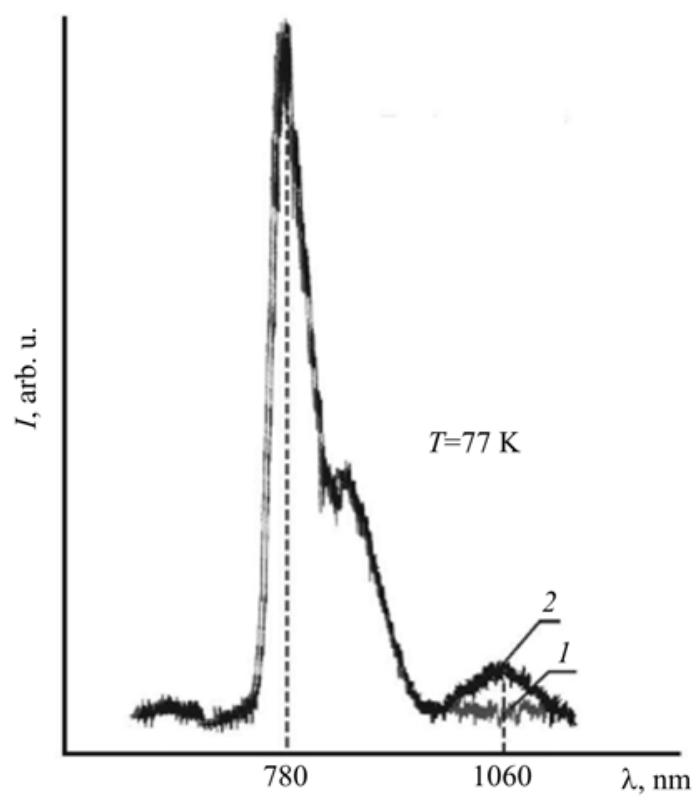

Fig. 5. Photoluminescence spectra of the undoped (curve 1) and Ag doped (curve 2) re-sintered CdTe layers; the spectra are measured at $77 \mathrm{~K}$.

parameters that determine the potentialities of using these layers in solar cell fabrication. Those parameters were obtained by van der Pauw and thermoelectromotive force methods, respectively. To carry out the measurements, the commercial Pt-Ag paste has been applied to the surface of the re-sintered layers.

It was found that CdTe layers prepared under optimal technological conditions have the $p$-type electrical conductivity and their resistivity is about $10^{5}$ to $10^{6} \mathrm{Ohm} \cdot \mathrm{cm}$. Those values are typical for screenprinted CdTe layers intended for solar cell fabrication.

To control the process of layer doping, photoluminescence spectra of the layers have been recorded. The impurity peak in the photoluminescence spectrum is indicative of $\mathrm{Ag}$ or $\mathrm{Au}$ impurity in the $\mathrm{CdTe}$ layer. Fig. 5 presents the photoluminescence spectra of undoped (curve 1) and Ag doped (curve 2) CdTe layers. As seen, the impurity peak whose position corresponds to the acceptor impurity $(\mathrm{Ag})$ is recorded in the photoluminescence spectrum of the doped layer. This peak lacks in the spectrum of the undoped CdTe layer.

\section{Conclusions}

As a result of annealing the screen-printed CdTe thick films in the inert gas medium, mechanically proof layers up to $80 \mathrm{~cm}^{2}$ in size, grain size up to $40 \mu \mathrm{m}$ and a resistivity no more than $10^{6} \mathrm{Ohm} \cdot \mathrm{cm}$ are obtained. The method for a direct synthesis of tellurides of some metals is developed. Ag and Au tellurides prepared by this method were used as doping materials for doping CdTe layers in the course of their re-sintering. By means 
of current studying the optical and electrophysical parameters of layers fabricated under different technological conditions, the optimal regimes of layer preparation are established. When developing the technology of fabrication of CdTe layers with required parameters, the electrical resistivity and luminescence spectra of the layers have been measured, morphology of the layer surface has been studied by the method of optical and atomic force microscopy, and the layer structure has been examined by the method of X-ray diffraction. Those layers are intended to be used in subsequent fabrication of solar cells based on $\mathrm{CdS} / \mathrm{CdTe}$ heterostructures.

\section{Acknowledgments}

Financial support for this work came through STCU project \# 1088.

\section{References}

1. D. Bonnet, H. Rabenhorst // In: 9th IEEE Photovoltaic Specialists Conf., IEEE, Silver Springs, MD, p.219 (1972).

2. S.S. Gorelik and M.Ya. Dashewcky, Material science of semiconductors and dielectrics. Moscow, Metallurgy (1988) (in Russian).

3. N. Nakayama, H. Matsumoto, K. Yamaguchi, S. Ikegami, and Y. Hioki // Jpn J. Appl. Phys. 15, N11, p.2281 (1976)

4. V.P. Kladko, O.S. Lytvyn, P.M. Lytvyn, et al. // Semiconductor Physics, Quantum Electronics and Optoelectronics 5, N 2, p. 170-175 (2002).

5. Handbuch: Der Praparativen Anorganischen Chemie. Herausgegeben von G. Brauer. Ferdinand Enke Verlag, Stuttgart (1956).

6. B.M. Bulakh, G.S. Pekar, Heat treatment of CdS large single crystals under controlled conditions // Kristall und Technik H. 5, B. 1, S. K15-K17 (1970).

7. L.J. van der Pauw, A method of measuring specific resistivity and Hall effect of discs of arbitrary shape // Phillips Res. Repts 13, N 1, p.1-9 (1958).

8. K. Kuribayashi, H. Matsumoto, H. Uda, et al. // Jpn J. Appl. Phys. 22, N12, p.1828-1831 (1983). 